TRANSACTIONS OF THE

AMERICAN MATHEMATICAL SOCIETY

Volume 356, Number 7 , Pages 2739-2756

S 0002-9947(03)03340-3

Article electronically published on October 21, 2003

\title{
EFFECT OF AGGREGATION ON POPULATION RECOVERY MODELED BY A FORWARD-BACKWARD PSEUDOPARABOLIC EQUATION
}

\author{
VÍCTOR PADRÓN
}

\begin{abstract}
In this paper we study the equation

$$
u_{t}=\Delta\left(\phi(u)-\lambda f(u)+\lambda u_{t}\right)+f(u)
$$

in a bounded domain of $\mathbb{R}^{d}, d \geq 1$, with homogeneous boundary conditions of the Neumann type, as a model of aggregating population with a migration rate determined by $\phi$, and total birth and mortality rates characterized by $f$. We will show that the aggregating mechanism induced by $\phi(u)$ allows the survival of a species in danger of extinction. Numerical simulations suggest that the solutions stabilize asymptotically in time to a not necessarily homogeneous stationary solution. This is shown to be the case for a particular version of the function $\phi(u)$.
\end{abstract}

\section{INTRODUCTION}

In this paper we will study the equation

$$
u_{t}=\Delta\left(\phi(u)-\lambda f(u)+\lambda u_{t}\right)+f(u), x \in \Omega, t \geq 0,
$$

with boundary conditions

$$
\eta \cdot \nabla\left(\phi(u)-\lambda f(u)+\lambda u_{t}\right)=0, x \in \partial \Omega, t \geq 0 .
$$

Here, $\Omega$ is a bounded domain in $\mathbb{R}^{d}, d \geq 1$, with regular boundary $\partial \Omega ; \eta$ is an exterior normal vector on the boundary $\partial \Omega$ of $\Omega ; \lambda>0$ is a constant. The functions $\phi$ and $f$ satisfy the following hypothesis:

Hypothesis 1. (1) $\phi$ is locally Lipschitz continuous, bounded and nonnegative.

(2) There exist $0<\alpha<\beta \leq+\infty$ such that $\phi$ is strictly increasing in $(0, \alpha)$, strictly decreasing in $(\alpha, \beta)$ and increasing, but not necessarily strictly increasing, in $[\beta,+\infty]$.

(3) $\phi(0)=0, \phi^{\prime}(0) \neq 0$.

(4) $f$ is locally Lipschitz continuous.

(5) There exist $0<a<b<\infty$ such that $f$ is negative in $(0, a) \cup(b,+\infty)$ and positive in $(a, b)$.

(6) $f(0)=0$ and $\lim \sup _{u \rightarrow \infty}|\phi(u)-\lambda f(u)|>\phi_{+}:=\phi(\alpha)$.

Received by the editors May 6, 2002 and, in revised form, January 22, 2003.

2000 Mathematics Subject Classification. Primary 35K70; Secondary 35R25, 92D25.

Key words and phrases. Pseudoparabolic equation, aggregating populations, recovery.

This research was supported in part by Consejo de Desarrollo Científico, Humanístico y Técnico (CDCHT) of the Universidad de Los Andes. 
(a)

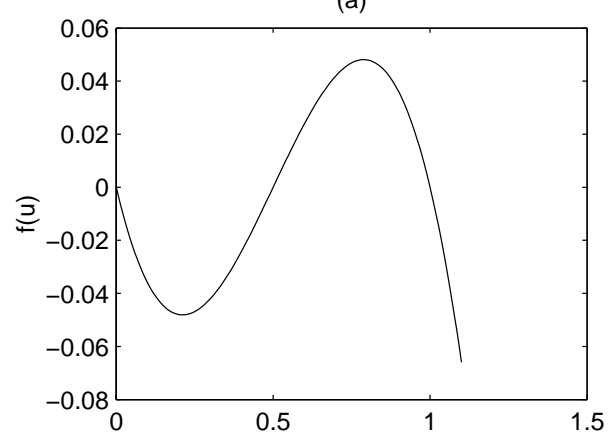

(c)

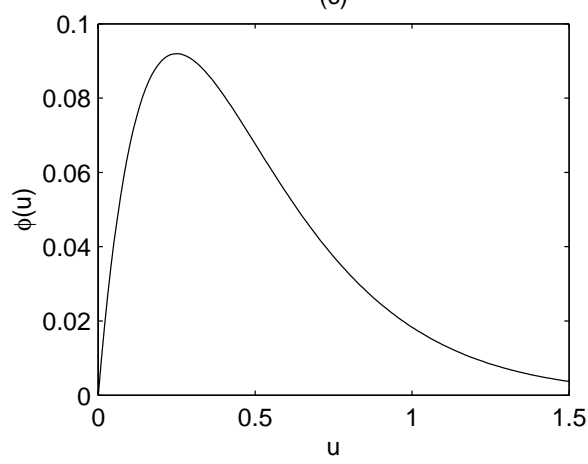

(b)

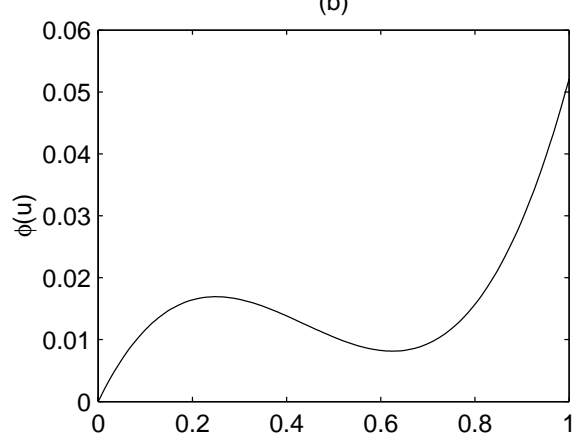

(d)

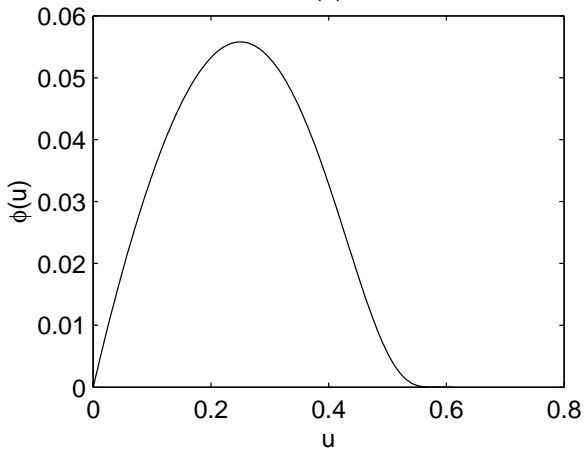

Figure 1. (a) $f(u)=u(u-a)(b-u)$, with $a=1 / 2$ and $b=1$; (b) $\phi(u)=\left(u^{3} / 3-(\alpha+\beta) u^{2} / 2+\alpha \beta u\right)$, with $\alpha=1 / 4$ and $\beta=5 / 8$; (c) $\phi(u)=u e^{-\frac{u}{\alpha}}$, with $\alpha=1 / 4 ;$ (d) $\phi(u)=u \exp \frac{(\beta-\alpha)^{2}}{\alpha(u-\beta)}$ if $0 \leq u<\beta$ and $\phi(u)=0$ if $u \geq \beta$, with $\alpha=1 / 4$ and $\beta=5 / 8$.

For example, the following functions are admissible: $f(u)=u(u-a)(b-u)$ where $a, b$ are constants such that $0<a<b ; \phi(u)=\left(u^{3} / 3-(\alpha+\beta) u^{2} / 2+\alpha \beta u\right)$ where $\alpha, \beta$ are constants such that $0<\alpha<\beta<3 \alpha ; \phi(u)=u e^{-\frac{u}{\alpha}}$ where $\alpha>0$ is a constant; and $\phi(u)=u \exp \frac{(\beta-\alpha)^{2}}{\alpha(u-\beta)}$ if $0 \leq u<\beta$ and $\phi(u)=0$ if $u \geq \beta$, where $\alpha, \beta$ are constants such that $0<\alpha<\beta$. See Figure 1 for a graphical representation of some of these functions.

This problem arises as a model for populations with the tendency to form groups. In this case $u(x, t)$ represents the population density for $x \in \Omega$, at the time $t \geq 0$; $\phi(u)=u \varphi(u)$ and $f(u)=u \sigma(u)$, where $\varphi(u)$ is the migration rate and $\sigma(u)$ is the net rate of population supply given by birth and death. Since we are mainly interested in aggregating populations, we will assume that $\varphi(u)$ is decreasing; that is, the migration of individuals from their present location is bigger if the population in this location is relatively small, and it is smaller if the population is high. The reproduction rate $\sigma(u)$ is of the logistic type with a threshold, that is, it satisfies the Allee effect. The Allee effect was originally proposed by W.C. Allee [1] to model the reduction of reproductive opportunities at low population densities. A population may exhibit Allee effects for a variety of reasons; see M.A. Lewis and P. Kareiva [8] 
and the literature therein. The main feature of the Allee effect is that below a threshold value the death rate is higher than the birth rate because, on average, the individuals cannot reproduce successfully. This, in turn, imposes a threat to the survival of the species at low densities.

We will show in this paper that the density dependent aggregating model (1.1)(1.2) contains mechanisms for the survival of a population menaced to extinction by the Allee effect. In fact, we will give conditions for a population located in a neighborhood of low density, to cluster together in an attempt to raise their local population above the threshold at which birth rate begins to exceed death rate. We give the name of recovery, properly defined below, to this kind of behavior.

The concept of recovery, first used without this name by P. Grindrod [5], was introduced by M. Lizana and V. Padrón [9] to study this type of phenomena and can also be seen as a measure of capability of a model to produce aggregation.

Extensive literature exists examining the role of diffusion in biological models: D.G. Aronson 2, 3], M.E. Gurtin \& R.C. MacCamy 7], J.D. Murray [10, A. Okubo [12], J.G. Skellan [15, 16], etc. However, phenomena such as insect swarming, fish schooling, animal grouping, and splitting and subsequent reamalgamation of herds are relatively common forms of behavior. In these cases there is a factor operating in the population which counteracts against diffusion and encourages individuals to aggregate; see A. Okubo [12, Ch. 7, pp. 110-131], where the ecological significance of grouping is discussed. Other models for aggregating populations, different from the one considered in this paper, that are based on partial differential equations, have been studied in the literature. We recommend the article of D. Grunbaum and A. Okubo [6] and the numerous references therein.

Problem (1.1) -(1.2) with $f(u) \equiv 0$ was studied by A. Novick-Cohen and R.L. Pego [11] and by V. Padrón [13]. A finite-dimensional model analogous to (1.1)(1.2) was studied by M. Lizana and V. Padrón in [9].

Density dependent models of population dynamics are usually based on equations of the form

$$
u_{t}=\Delta \phi(u)+f(u) .
$$

In deriving (1.3) it is assumed that individuals disperse to avoid crowding (see Gurtin \& MacCamy [7]). Note that, in general, $\phi^{\prime}(0)=0$ so that (1.3) is a degenerate parabolic equation. However, if $\phi^{\prime}(u)>0$ for $u>0$, then equation (1.3) can be dealt with by means of existing theory (Aronson [3], Gurtin \& MacCamy [7]). In the case of aggregating populations, $\phi^{\prime}(u)$ may be negative for positive values of $u$; therefore, the standard initial boundary value problems for (1.3) are ill posed.

One possibility to overcome this difficulty is to substitute (1.3) by

$$
u_{t}=\Delta J+f(u)
$$

where $J(x, t)$ is the average

$$
J(x, t):=\int_{\Omega} K(x, y) \phi(u(y, t)) d y,
$$

with a nonnegative $K(x, y)$. A particular convenient choice (see P. Grindrod [5]) is to define $K(x, y)$ as the Green function of $(I-\lambda \Delta)$, for a constant $\lambda>0$, in the domain $\Omega$. We can also impose no-flux boundary conditions on $J$ to assure the isolation of $\Omega$. 
That is, for each $t \geq 0, J(x, t)$ is the solution to the problem

$$
\left\{\begin{aligned}
(I-\lambda \Delta) J(x, t) & =\phi(u(x, t)), x \in \Omega, \\
\eta \cdot \nabla J & =0, x \in \partial \Omega .
\end{aligned}\right.
$$

In $\S 2$ of this paper we will show (Proposition 3) that problem (1.4)-(1.5) is equivalent to (1.1)-(1.2). Also in this section we will show the existence and uniqueness of the solutions of (1.1)-(1.2) globally defined for all $t \geq 0$, and we will obtain some results related to the regularity of the solutions.

In $\S 3$, we will show that the aggregating mechanism that induces $\phi(u)$, in the regions where $\phi^{\prime}(u)<0$, allows for the survival of a species in danger of extinction due to its low population density. In this section we show some numerical simulations that suggest that the solutions stabilize asymptotically in time to a not necessarily homogeneous stationary solution. In $\S 4$ we will show that this is the case when $\phi(u) \equiv 0$ for $u \geq a$. We will show that the solutions of (1.1)-(1.2) converge pointwise to a stationary solution $u_{\infty}(x)$, not necessarily homogeneous, as $t \rightarrow \infty$. In this section we will also show that there are populations located below the threshold value $u=a$ that overcome this difficulty by aggregating to increase their population density to the region $(a, b)$ where the net rate of population supply becomes positive. In fact, in the last result of this paper we will prove that these populations stabilize to a discontinuous steady state solution $u_{\infty}(x)$ of the type

$$
u_{\infty}(x)=\left\{\begin{array}{lll}
b & \text { if } & x \in \Omega, \\
0 & \text { if } \quad x \in \Omega \backslash \Omega_{0},
\end{array}\right.
$$

where $\Omega_{0}$ is a subset of $\Omega$ of positive measure.

\section{EXISTENCE AND UNIQUENESS OF SOlUtions}

In this section we will show a result concerning the existence and uniqueness of solutions of (1.1) -(1.2) globally defined in time. We also show certain regularity properties, and a useful representation of the solution by a parameterized ODE.

First we will reformulate problem (1.1)-(1.2) as

$$
u_{t}=\lambda^{-1}\left[(I-\lambda \Delta)^{-1}-I\right] \phi(u)+f(u)
$$

where the operator $(I-\lambda \Delta)^{-1}$ is defined by setting $h=(I-\lambda \Delta)^{-1} k$. Here $h$ is the solution to the problem

$$
\left\{\begin{aligned}
(I-\lambda \Delta) h(x) & =k(x), x \in \Omega, \\
\eta \cdot \nabla h & =0, x \in \partial \Omega .
\end{aligned}\right.
$$

Now we show the existence and uniqueness of local solutions for problem (2.1). Later we will show, in Proposition 3, that the solutions of (2.1) are precisely the solutions of (1.1)-(1.2).

Theorem 1 (Local Existence). Suppose that $\phi$ and $f$ are locally Lipschitz and that $u_{0} \in X$, where $X$ is the space $L_{\infty}(\Omega)$ or $C(\bar{\Omega})$. Then, there exists $T>0$ such that the equation (2.1) has a unique solution $u(t)$ with $u(t) \in C^{1}([0, T], X)$ and $u(0)=u_{0}$. If $\phi$ and $f$ are $C^{m}, 1 \leq m \leq \infty$, then $u \in C^{m+1}([0, T], X)$. If $\phi$ and $f$ are analytic, then $u \in H([0, T], X)$, the space of analytic functions with values in $X$. 
Proof. We will regard (2.1) as an ODE on the Banach space $X$. Theorem 1 will follow from standard results for such ODEs (e.g. see [4]). We need only to verify that the map

$$
u \rightarrow \psi(u):=\lambda^{-1}\left[(I-\lambda \Delta)^{-1}-I\right] \phi(u)+f(u)
$$

is locally Lipschitz, $C^{k}$, or analytic in $X$ if $\psi$ is. But this is true when the map

$$
k \rightarrow(I-\lambda \Delta)^{-1} k
$$

is bounded in $X$. This follows from the following lemma, the proof of which is given in [11, Lemma 2.2].

Lemma 2. Let $k \in L_{\infty}(\Omega)$. Then, problem (2.2) has a unique solution $h$ that is in $W^{2, p}(\Omega)$ for all $p$ such that $1<p<\infty$. If $k(x)$ is not a constant almost everywhere, then

$$
\underset{\Omega}{\operatorname{essinf}} k(\cdot)<h(x)<\underset{\Omega}{\operatorname{ess} \sup } k(\cdot)
$$

for all $x \in \Omega$.

Remark 1. In the same way as in Theorem 1) we can prove that (2.1) has a local solution defined in an interval of the form $(-T, 0]$. We can do this by inverting the direction of time in (2.1) with the change of variable $\tau=-t$ and solving the resulting problem. Therefore, we can assume that (2.1) has a unique solution defined in an interval of the form $[S, T]$ with $S<0$ and $T>0$.

Proposition 3. The solution $u(t)$ of Theorem 1 is the unique solution of (1.1)(1.2).

Proof. Let us define

$$
J(x, t):=\phi(u)-\lambda f(u)+\lambda u_{t} .
$$

Then, from (2.1) we have

$$
J=(I-\lambda \Delta)^{-1} \phi(u) .
$$

Therefore $J \in C\left(\left(T_{1}, T_{2}\right), W^{2, p}(\Omega)\right)$ for $1<p<\infty, \eta \cdot \nabla J=0$ in $\partial \Omega$, and

$$
\Delta J=\lambda^{-1}(\lambda \Delta-I+I)(I-\lambda \Delta)^{-1} \phi(u)=u_{t}-f(u) .
$$

Hence, equation (1.1) is satisfied. Conversely, if $u(x, t)$ is a solution of (1.1)-(1.2),

$$
u_{t}=\Delta\left(\phi(u)-\lambda f(u)+\lambda u_{t}\right)+f(u) .
$$

Therefore,

$$
\begin{aligned}
(I-\lambda \Delta)^{-1} u_{t} & =(I-\lambda \Delta)^{-1} \Delta\left(\phi(u)-\lambda f(u)+\lambda u_{t}\right)+(I-\lambda \Delta)^{-1} f(u) \\
& =\Delta(I-\lambda \Delta)^{-1}\left(\phi(u)-\lambda f(u)+\lambda u_{t}\right)+(I-\lambda \Delta)^{-1} f(u) \\
& =\Delta(I-\lambda \Delta)^{-1}\left(\phi(u)-\lambda \Delta(I-\lambda \Delta)^{-1} f(u)+\lambda \Delta(I-\lambda \Delta)^{-1} u_{t}\right. \\
& +(I-\lambda \Delta)^{-1} f(u) .
\end{aligned}
$$

Hence,

$$
(I-\lambda \Delta)(I-\lambda \Delta)^{-1} u_{t}=\Delta(I-\lambda \Delta)^{-1} \phi(u)+(I-\lambda \Delta)(I-\lambda \Delta)^{-1} f(u) .
$$

That is,

$$
u_{t}=\Delta(I-\lambda \Delta)^{-1} \phi(u)+f(u) .
$$

This shows that $u$ is a solution of (2.1). Therefore, it is unique. 
The following lemma, the proof of which is similar to [11, Lemma 2.5], will be useful in the sequel. It will allow us to regard the solutions $u(x, t)$ of (1.1) -(1.2) for almost all $x$ as a classical solution of the ordinary differential equation (2.3).

Let $B(\Omega)$ be the space of functions in $\Omega$ that are bounded in the sup-norm. Recall that $L_{\infty}(\Omega)$ is the space of equivalence classes of functions in $B(\Omega)$ that differ on a set of measure zero.

Lemma 4. Let $u(t)$ be the solution of (1.1)-(1.2) in $L_{\infty}(\Omega)$ given by Theorem 1 , defined in a maximal interval $(S, T)$. Given any representative $u_{0 *}$ of the equivalence class $u_{0}$, there exists a set $\Omega_{*} \subset \Omega$ such that $\Omega \backslash \Omega_{*}$ has measure zero, and a function $u_{*} \in C((S, T), B(\Omega))$, with $u_{*}(t) \in u(t)$ for all $t \in(S, T)$, such that if $x \in \Omega_{*}$, then the function $t \rightarrow u_{*}(x, t)$ is $C^{1}$ in $(S, T)$ and is a classical solution of the ordinary differential equation

$$
\lambda \frac{d u(t)}{d t}=\lambda f(u(t))-\phi(u(t))+J(x, t) .
$$

Next we will establish a result about the regularity of the solutions of (1.1)-(1.2).

Proposition 5 (Regularity). Let $u(t)$ be the solution of (1.1)-(1.2) obtained by Theorem 1 and defined in a maximal interval $(S, T)$. Let $t \in(S, T)$ and fix $x \in \Omega_{*}$. Then,

If $u_{0}$ is continuous in $x$, then $u(\cdot, t)$ is continuous in $x$.

If $u_{0}$ is not continuous in $x$, then $u(\cdot, t)$ is not continuous in $x$.

Proof. Regarding $x$ as a parameter in equation (2.4), this result follows from standard results on continuous dependence on initial values for solutions of ODEs. Continuity at time 0 implies continuity at time $t$ and vice versa.

Now we describe some positively invariant regions for solutions of (1.1)-(1.2).

A set $U \subset \mathbb{R}$ is positively invariant for the problem (1.1)-(1.2) if for $u_{0}(x) \in U$ and all $x \in \Omega_{*}$, we have $u(x, t) \in U$ for all $t \geq 0$.

Proposition 6 (Positively Invariant Regions). 1) Let $M$ be such that $(\phi-\lambda f)(M)$ $>\phi_{+}$; then $U=[0, M)$ is positively invariant. In particular, if $\alpha<a, U=[0, \alpha)$ is positively invariant.

2) If $\beta=a$ and $\phi(u)=0$ for all $u \geq a$, then $U=(a,+\infty)$ and $U_{\epsilon}=[b-\epsilon, b+\epsilon]$, with $\epsilon<b-a$, are positively invariant.

Proof. Let $u(x, t)$ be a solution of (1.1)-1.2) with $u(x, 0)=u_{0}(x)$ defined on a maximal interval $(S, T)$.

From (2.1) we obtain, if $J(x, t):=\phi(u(x, t))-\lambda f(u(x, t))+u_{t}(x, t)$, that

$$
J=(I-\lambda \Delta)^{-1} \phi(u(x, t)) \text {. }
$$

Then, since $u(\cdot, t) \in L_{\infty}(\Omega)$, we have by Lemma 2 that for $u(x, t) \not \equiv$ constant

$$
\underset{\Omega}{\operatorname{essinf}} \phi(u(\cdot, t))<J(x, t)<\underset{\Omega}{\operatorname{ess} \sup } \phi(u(\cdot, t))
$$

for all $x \in \Omega$ and all $t \in(S, T)$.

Moreover, from Lemma 4 we know that there exists $\Omega_{*}$ such that the measure of $\Omega \backslash \Omega_{*}$ is zero and that for all $x \in \Omega_{*}$ the function $t \rightarrow u(x, t)$ is $C^{1}$ on $(S, T)$ and is a classical solution of the ODE

$$
\lambda \frac{d u}{d t}=J(x, t)-[\phi(u)-\lambda f(u)] .
$$


Now, suppose that $u_{0}(x) \in U=[0, M)$ for all $x \in \Omega_{*}$.

If $u_{0}(x) \equiv$ constant, it can be shown that for all $x \in \Omega, u(t):=u(x, t)$ is a solution of the ODE

$$
\frac{d u}{d t}=f(u)
$$

in the interval $(S, T)$. From this we obtain that $U=[0, M)$ is positively invariant.

Suppose now that $u_{0}(x) \not \equiv$ constant and that $u_{0}(x) \in U=[0, M)$ for all $x \in \Omega_{*}$. Let $u\left(x_{0}, t_{0}\right)=0$ for $t_{0} \geq 0, x_{0} \in \Omega_{*}$ such that $0 \leq u\left(x, t_{0}\right) \leq M$ for all $x \in \Omega_{*}$. Since

$$
0<J\left(x, t_{0}\right)<\underset{\Omega}{\operatorname{ess} \sup } \phi\left(u\left(\cdot, t_{0}\right)\right) \leq \phi_{+}
$$

for all $x \in \Omega$, we obtain, for $x \in \Omega_{*}$ and $t \in(S, T)$, that

$$
\lambda \frac{d u}{d t}\left(x_{0}, t_{0}\right)=J\left(x_{0}, t_{0}\right)-\left[\phi\left(u\left(x_{0}, t_{0}\right)\right)-\lambda f\left(u\left(x_{0}, t_{0}\right)\right)\right]=J\left(x_{0}, t_{0}\right)>0 .
$$

This proves that $u(x, t) \geq 0$ for all $x \in \Omega_{*}$ and $t \geq 0$.

Suppose now, by contradiction, that there exist $t_{0}>0$ and $x_{0} \in \Omega_{*}$ such that $u\left(x_{0}, t_{0}\right)=M$.

Hence,

$$
\begin{aligned}
\lambda \frac{d u}{d t}\left(x_{0}, t_{0}\right) & =J\left(x_{0}, t_{0}\right)-\left[\phi\left(u\left(x_{0}, t_{0}\right)\right)-\lambda f\left(u\left(x_{0}, t_{0}\right)\right)\right] \\
& =J\left(x_{0}, t_{0}\right)-[\phi-\lambda f](M) \\
& <\phi_{+}-\phi_{+}=0 .
\end{aligned}
$$

This contradicts the fact that $0 \leq u_{0}(x)<M$ for all $x \in \Omega_{*}$. This completes the proof of 1 ).

Suppose now that $\beta=a, \phi(u)=0$ for $u \geq a$ and that $u_{0}(x) \in(a,+\infty)$ for all $x \in \Omega_{*}$. If $u_{0}(x) \equiv$ constant, the result is obtained from the fact that $u(t):=u(x, t)$ satisfies the equation $\frac{d u(t)}{d t}=f(u(t))$. Suppose then that $u_{0}(x) \not \equiv$ constant; in this case we know that for all $x \in \Omega, u(x, t)$ satisfies the equation

$$
\lambda \frac{d u}{d t}=J(x, t)-\phi(u)+\lambda f(u)
$$

Here $J(x, t)>0$. Suppose that there exist $t_{0}>0$ and $x_{0} \in \Omega_{*}$ such that $u\left(x_{0}, t_{0}\right)=a$ and $u\left(x_{0}, t\right)>a$ for $0 \leq t<t_{0}$. Then, from (2.5) we obtain that

$$
\lambda \frac{d u}{d t}\left(x_{0}, t_{0}\right)=J\left(x_{0}, t_{0}\right)>0,
$$

which is a contradiction. This proves the first part of $\mathbf{2}$ ).

The second part of 2) follows from the fact that all solutions of (1.1)-(1.2) that satisfy $u(x, t) \geq a$ for all $x \in \Omega_{*}$ and all $t$ in an interval $I$ of $\mathbb{R}$, satisfy the ODE

$$
\frac{d u}{d t}(t)=f(u(t))
$$

on $I$. This follows from the fact that if $u(x, t) \geq a$ for all $x \in \Omega_{*}$, then $\phi(u(x, t)) \equiv 0$ on $\Omega_{*}$. Moreover, since $J(x, t)$ is a solution of the equation $\Delta w=\phi(u(x, t))$, this implies that $J(x, t) \equiv 0$ on $\Omega_{*}$, and we obtain 2).

Remark 2. Notice that part 1) of Proposition 6 allows us to obtain an a priori bound for the solutions of (1.1)-(1.2). 
Theorem 7 (Global Existence). If $u_{0} \in X$, where $X=L_{\infty}(\Omega)$ or $C(\bar{\Omega})$, then there exists a unique solution $u(x, t)$ of (1.1) -(1.2) with initial data $u_{0}$ such that $u \in C^{1}([0, \infty), X)$.

Proof. The global existence is obtained by standard results on ODEs given the a priori bound obtained by part 1) of Proposition [6 for the norm $\|u(t)\|_{L_{\infty}}$ of the solutions.

\section{RECOVERY PROPERTY}

In this section we will give conditions on $\phi$ and $f$ that allow a population located in a neighborhood of low density to cluster together in an attempt to raise their local population above the threshold at which the birth rate begins to exceed the death rate. We give the name of recovery to this property.

Definition 1. We will say that problem (1.1)-(1.2) exhibits recovery if there exists a solution $u(x, t)$ of (1.1)-(1.2) with $0 \leq u(x, 0)<a$ for all $x \in \Omega$, such that for some $t_{0}>0$ and $\Omega_{0} \subset \Omega$ of positive measure, $u\left(x, t_{0}\right)>a$ for almost all $x \in \Omega_{0}$. We will say that the recovery is permanent if $u(x, t)>a$ for all $t>t_{0}$ and almost every $x \in \Omega_{0}$.

The following result gives a necessary and sufficient condition for problem (1.1)(1.2) to exhibit recovery.

Let $\gamma:=\inf A_{\alpha}$ if $A_{\alpha} \neq \emptyset$ and $\gamma:=+\infty$ if $A_{\alpha}=\emptyset$, where $A_{\alpha}:=\{u>0: \phi(u)>$ $\left.\phi(\alpha):=\phi_{+}\right\}$.

Proposition 8. Problem (1.1)-(1.2) exhibits recovery if and only if $\alpha<a<\gamma$.

Proof. Suppose first that problem (1.1) -(1.2) exhibits recovery but nevertheless $a \leq \alpha$ or $\gamma \leq a$. Here we assume that $\gamma<+\infty$ in the case that $\gamma \leq a$. This implies that there exist a first value $t_{0}^{*}>0$ and $x_{0} \in \Omega^{*} \operatorname{such}$ that $\operatorname{ess}_{\sup _{\Omega}} u\left(\cdot, t_{0}^{*}\right) \leq$ $u\left(x_{0}, t_{0}^{*}\right)=a$. It is obvious that in this case $\frac{\partial u}{\partial t}\left(x_{0}, t_{0}^{*}\right) \geq 0$.

Since $u \equiv a$ is an equilibrium solution of (1.1)-(1.2), we can assure that $u\left(x, t_{0}^{*}\right)<$ $a$ for all $x$ in a subset of positive measure.

Since we assume that $a \leq \alpha$ or $\gamma \leq a$, we have

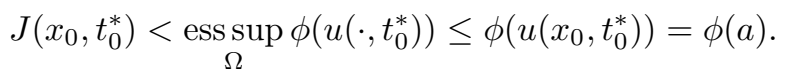

Therefore,

$$
\lambda u_{t}\left(x_{0}, t_{0}^{*}\right)=\lambda f(a)-\phi(a)+J\left(x_{0}, t_{0}^{*}\right)<0,
$$

and this is a contradiction.

Conversely, suppose that $\alpha<a<\gamma$ and let $u(x, t)$ be a solution of (1.1)-(1.2) such that $u(x, 0)=a$ for $x \in \Omega_{0}$, and $u(x, 0)=\alpha$ if $x \in \Omega \backslash \Omega_{0}$, where $\Omega_{0}$ is a subset of $\Omega$ of positive measure.

Since

$$
\phi(a)<J(x, 0)<\phi(\alpha),
$$

we have that

$$
\lambda u_{t}(x, 0)=\lambda f(a)-\phi(a)+J(x, 0)>0,
$$


TABle 1. Numerical values of the solution $u(x, t)$ of (1.1)-1.2 with $\Omega=(0,1), \lambda=.05, \phi(u)=9\left(\frac{u^{3}}{3}-\frac{59 u^{2}}{120}+\frac{11 u}{60}\right), f(u)=$ $.36 u(u-.5)(1-u)$, and initial data $u_{0}(x)=\frac{29}{60} e^{-\frac{(x-.5)^{2}}{.26-(x-.5)^{2}}}$.

\begin{tabular}{c|ccc}
\hline \hline $\mathrm{t} \backslash \mathrm{x}$ & .08 & .18 & .28 \\
\hline \hline 0 & 0.05859554676105 & 0.25238617471082 & 0.38451126541657 \\
10 & 0.03026187118585 & 0.03064667102072 & 0.03132217672434 \\
20 & 0.03025810169809 & 0.03064288101552 & 0.03131835069636 \\
30 & 0.03025810131471 & 0.03064288063004 & 0.03131835030722 \\
40 & 0.03025810131468 & 0.03064288063002 & 0.03131835030719 \\
50 & 0.03025810131468 & 0.03064288063002 & 0.03131835030719 \\
60 & 0.03025810131468 & 0.03064288063002 & 0.03131835030719 \\
70 & 0.03025810131468 & 0.03064288063002 & 0.03131835030719 \\
80 & 0.03025810131468 & 0.03064288063002 & 0.03131835030719 \\
90 & 0.03025810131468 & 0.03064288063002 & 0.03131835030719 \\
100 & 0.03025810131468 & 0.03064288063002 & 0.03131835030719 \\
\hline \hline $\mathrm{t} \backslash \mathrm{x}$ & & & .48 \\
\hline \hline 0 & 0.45580934769276 & 0.48258917130442 & \\
10 & 0.86415065391787 & 0.86534431914688 & \\
20 & 0.86414295318602 & 0.86533667442184 & \\
30 & 0.86414295240276 & 0.86533667364427 & \\
40 & 0.86414295240270 & 0.86533667364422 & \\
50 & 0.86414295240270 & 0.86533667364422 & \\
60 & 0.86414295240270 & 0.86533667364422 & \\
70 & 0.86414295240270 & 0.86533667364422 & \\
80 & 0.86414295240270 & 0.86533667364422 & \\
90 & 0.86414295240270 & 0.86533667364422 & \\
100 & 0.86414295240270 & 0.86533667364422 & \\
\hline \hline & & & \\
\hline
\end{tabular}

for all $x \in \Omega_{0}$. This, together with the continuity of the solutions of (1.1)-(1.2) with respect to the initial data and the fact that the solutions of (1.1)-(1.2) can be extended locally to $t<0$, proves our assertion.

Proposition 8 tells us that (1.1)-(1.2) exhibits recovery. This is illustrated in Figure 2 where the phase portrait of the solution $u(x, t)$ of (1.1)-(1.2) is plotted. Although $u(x, t)$ converges uniformly to zero as $t \rightarrow \infty$, we observe an almost immediate recovery from an initial data $u_{0}$ located below the threshold level $a=.5$, which is sustained for a relatively long interval of time.

The numerical experiments shown in Figure 3 and the corresponding data sequence recorded in Table 1 suggest that (1.1)-(1.2) also exhibits permanent recovery. Here we have chosen the same initial distribution as in Figure 2, but changed the value of $\beta$ in $\phi(u)$ from $\beta=2 / 3$ in Figure 2 to $\beta=11 / 15$ in Figure 3. Another instance of permanent recovery is shown in Figure 4 with the same equation as in Figure 3 but with different initial data.

In the next section we will prove, for a particular class of functions $\phi(u)$, that (1.1) - (1.2) exhibits permanent recovery. 


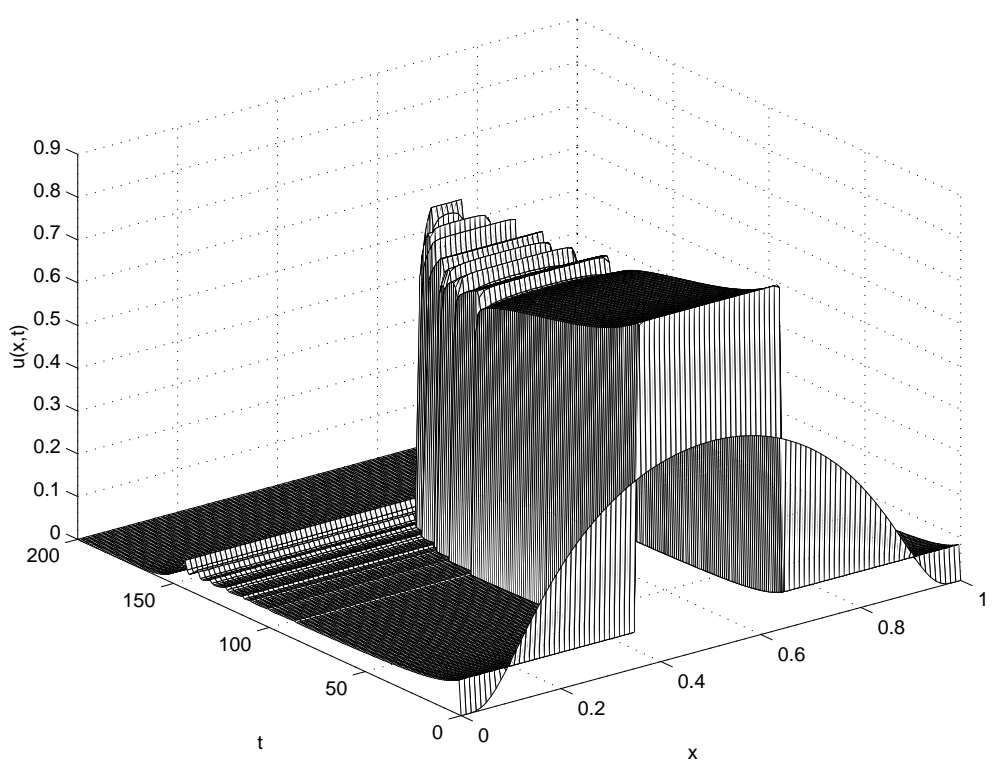

Figure 2. Phase portrait of the solution $u(x, t)$ of 1.1 - (1.2) with $\Omega=(0,1), \lambda=.05, \phi(u)=9\left(\frac{u^{3}}{3}-\frac{11 u^{2}}{24}+\frac{u}{6}\right), f(u)=$ $.36 u(u-.5)(1-u)$, and initial data $u_{0}(x)=\frac{29}{60} e^{-\frac{(x-.5)^{2}}{.26-(x-.5)^{2}}}$.

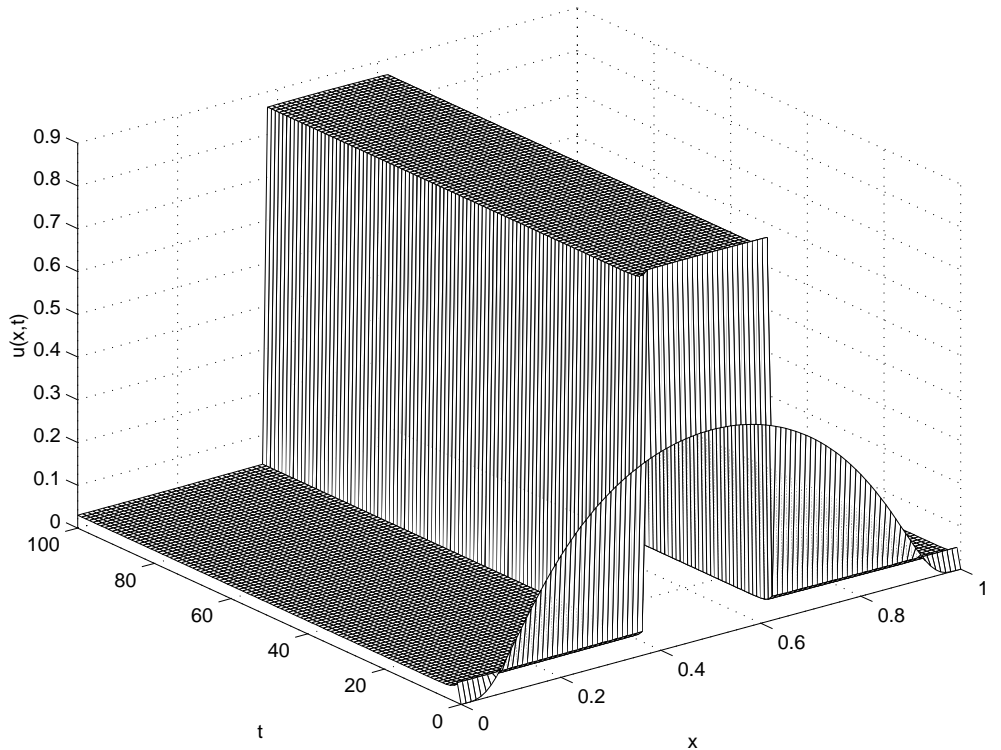

Figure 3. Phase portrait of the solution $u(x, t)$ of (1.1)-(1.2) with $\Omega=(0,1), \lambda=.05, \phi(u)=9\left(\frac{u^{3}}{3}-\frac{59 u^{2}}{120}+\frac{11 u}{60}\right), f(u)=$ $.36 u(u-.5)(1-u)$, and initial data $u_{0}(x)=\frac{29}{60} e^{-\frac{(x-.5)^{2}}{.26-(x-.5)^{2}}}$. 


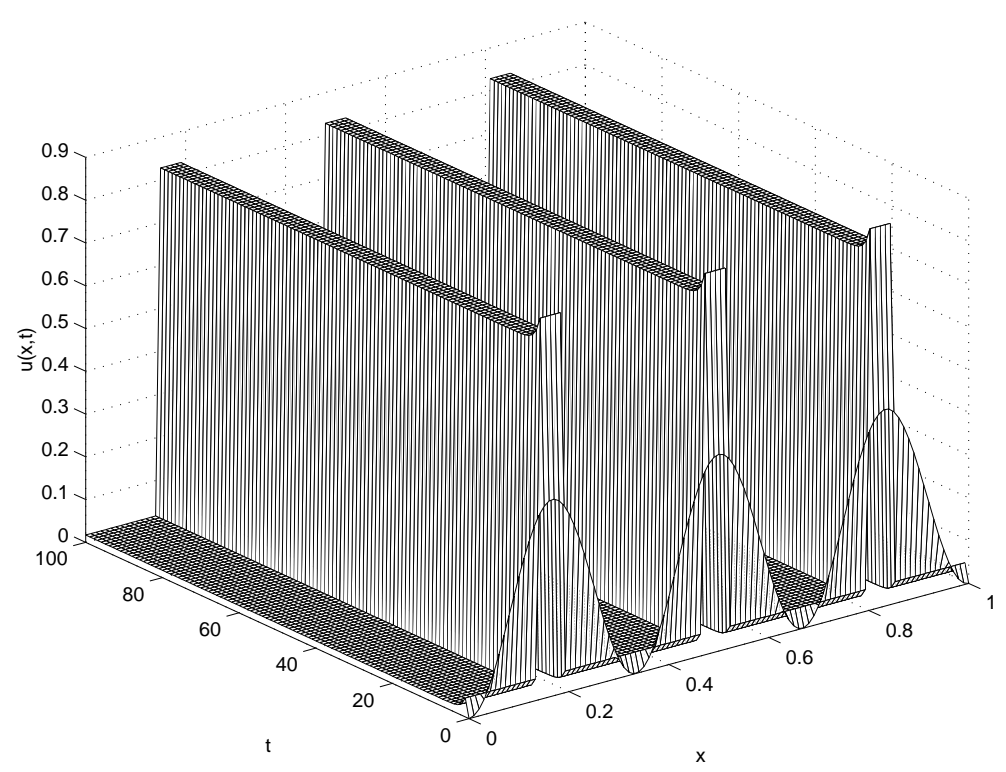

Figure 4. Phase portrait of the solution $u(x, t)$ of 1.1 - (1.2) with $\Omega=(0,1), \lambda=.05, \phi(u)=9\left(\frac{u^{3}}{3}-\frac{59 u^{2}}{120}+\frac{11 u}{60}\right), f(u)=$ $.36 u(u-.5)(1-u)$, and initial data $u_{0}(x)=.23\left(1+\sin \left(6 \pi x+\frac{3}{2} \pi\right)\right)$.

\section{Stabilization in a particular case}

In this section we study problem (1.1)-1.2) when $\beta=a$ and $\phi(u)=0$ for $u \geq a$. For example, this condition is satisfied by

$$
\phi(u)= \begin{cases}u \exp \frac{(a-\alpha)^{2}}{\alpha(u-a)} & \text { if } 0 \leq u<a, \\ 0 & \text { if } u \geq a\end{cases}
$$

with $0<\alpha<a$.

We will show that all solutions of (1.1)-(1.2) stabilize to a not necessarily homogeneous steady state solution.

We will begin with the following lemma which describes the dissipative nature of problem (1.1)-(1.2) in this particular case.

Lemma 9. Given a $C^{1}$ function $h: \mathbb{R} \rightarrow \mathbb{R}$ such that $h(0)=0$ and $h \geq 0$ on $[0, \infty)$, let $H(z):=\int_{0}^{z} h(\phi(s)) d s$. Let $u(x, t)$ be a solution of (1.1)-(1.2) defined for all $t \geq 0$.

1) If $h^{\prime}(z) \geq 0$ for all $z$, then $\int_{\Omega} H(u(x, t)) d x$ is a nonincreasing function of $t$ for $t \in[0, \infty)$.

2) $\lim _{t \rightarrow \infty} \int_{\Omega} H(u(x, t)) d x$ exists, independently of the sign of $h^{\prime}(z)$.

Proof. Let $h \in C^{1}$ be such that $h^{\prime}(z) \geq 0$ for all $z$. Then

$$
\begin{aligned}
\frac{d}{d t} \int_{\Omega} H(u(x, t)) d x & =\int_{\Omega} h(\phi(u)) u_{t} d x \\
& =\int_{\Omega} h(\phi(u))\left[u_{t}-f(u)\right] d x+\int_{\Omega} h(\phi(u)) f(u) d x
\end{aligned}
$$




$$
\begin{aligned}
= & -\int_{\Omega}[h(J)-h(\phi(u))]\left[u_{t}-f(u)\right] d x+\int_{\Omega} h(J)\left[u_{t}-f(u)\right] d x \\
& +\int_{\Omega} h(\phi(u)) f(u) d x \\
= & -\lambda \int_{\Omega} h^{\prime}(z(x, t))\left[u_{t}-f(u)\right]^{2} d x+\int_{\Omega} h(J) \Delta J d x \\
& +\int_{\Omega} h(\phi(u)) f(u) d x \\
= & -\lambda \int_{\Omega} h^{\prime}(z(x, t))\left[u_{t}-f(u)\right]^{2} d x+\int_{\Omega} \nabla \cdot(h(J) \nabla J) d x \\
& -\int_{\Omega} h^{\prime}(J)|\nabla J|^{2} d x+\int_{\Omega} h(\phi(u)) f(u) d x \\
= & -\lambda \int_{\Omega} h^{\prime}(z(x, t))\left[u_{t}-f(u)\right]^{2} d x-\int_{\Omega} h^{\prime}(J)|\nabla J|^{2} d x \\
& +\int_{\Omega} h(\phi(u)) f(u) d x \\
\leq & 0,
\end{aligned}
$$

from which we obtain 1). Now we consider part 2). When $h^{\prime}(z) \geq 0$, the result follows from the fact that $\int_{\Omega} H(u(x, t)) d x$ is decreasing and bounded below; in fact, since $u_{0}(x) \geq 0$, Proposition 6 implies that $\int_{\Omega} H(u(x, t)) d x \geq 0$.

If $h(z)$ is not increasing, let $k(z):=z+\epsilon h(z)$, with $\epsilon>0$ small enough such that $k^{\prime}(z) \geq 0$ for $|z| \leq \sup _{(x, t)}|\phi(u(x, t))|$. If $K^{\prime}(z)=k(\phi(z))$, and $\Phi^{\prime}(z)=\phi(z)$, then

$$
\epsilon \int_{\Omega} H(u)(t)=\int_{\Omega} K(u)(t)-\int_{\Omega} \Phi(u)(t)+C .
$$

As in part 1), the limit of the right hand side exists, and we obtain 2).

In the following result we will show, under a "nondegeneracy condition" on $\phi$, that the solutions of (1.1)-(1.2) converge pointwise to a stationary solution $u_{\infty}(x)$ as $t \rightarrow \infty$.

For $0<r<\phi_{+}$, denote by $u_{i}(r), i=1,2$, the two roots of $\phi(u)=r$ that satisfy

$$
\phi\left(u_{i}(r)\right)=r, \quad i=1,2, \quad u_{1}(r)<u_{2}(r) .
$$

Hypothesis 2. There are no nonnegative constants $\mu_{i}, i=0,1,2$, not all zero, such that $\sum_{i=1}^{2} \mu_{i} u_{i}(r) \equiv \mu_{0}$ independently of $r$ for all $r$ in any open subinterval of $\left(0, \phi_{+}\right)$.

Hypothesis 2 does not allow the existence of a continuum of steady states for problem (1.1)-(1.2) with $f \equiv 0$ (see [13] for a discussion on the justification of this hypothesis), and will be needed in the following theorem. It is not difficult to verify that Hypothesis 2 is satisfied by $\phi(u)$ given by (4.1).

Theorem 10. Suppose that $\phi$ and $f$ satisfy Hypothesis 1 with $\beta=a$ and $\phi(u)=0$ for $u \geq a$. Let $u_{0} \in L_{\infty}(\Omega)$ be such that $u_{0}(x) \geq 0$ for almost every $x$ in $\Omega$. Let $u(x, t)$ be a solution of (1.1)-(1.2) with initial data $u_{0}$, defined for all $t \geq 0$. If $\phi$ satisfies Hypothesis 圆, then

$$
u_{\infty}(x):=\lim _{t \rightarrow \infty} u(x, t)
$$

exists for almost every $x \in \Omega, \phi\left(u_{\infty}(x)\right)=$ constant, and $f\left(u_{\infty}(x)\right)=0$. 
Remark 3. The convergence of $u(x, t)$ is not necessarily uniform. See Proposition 5 and the comments previous to Proposition 14 at the end of this section.

Proof. The proof is divided into three lemmas. Let

$$
\phi_{p r}(t):=|\Omega|^{-1} \int_{\Omega} \phi(u(x, t)) d x=|\Omega|^{-1} \int_{\Omega} J(x, t) d x .
$$

Here $u(x, t)$ is the solution of (1.1)-(1.2) and $J(x, t)=\phi(u(x, t))-\lambda f(u(x, t))+$ $\lambda u_{t}(x, t)$.

Lemma 11. Under the hypotheses of Theorem 10, as $t \rightarrow \infty$ we have

$$
\begin{gathered}
\left\|u_{t}(\cdot, t)-f(u(\cdot, t))\right\|_{L_{2}} \rightarrow 0, \\
\left\|J(\cdot, t)-\phi_{p r}(t)\right\|_{L_{\infty}} \rightarrow 0, \\
\left\|\phi(u(\cdot, t))-\phi_{p r}(t)\right\|_{L_{2}} \rightarrow 0 .
\end{gathered}
$$

Proof. Property (4.4) is obtained from (4.2) and (4.3).

Let us choose a primitive $\Phi(z):=\int_{0}^{z} \phi(s) d s$. Then,

$$
\frac{d}{d t} \int_{\Omega} \Phi(u(x, t)) d x \leq-\int_{\Omega}\left[\lambda\left(u_{t}-f(u)\right)^{2}+|\nabla J|^{2}\right] d x
$$

Therefore, for all $T>0$ we have

$$
\lambda \int_{0}^{T} \int_{\Omega}\left(u_{t}-f(u)\right)^{2} d x d t+\int_{0}^{T} \int_{\Omega}|\nabla J|^{2} d e d t \leq \int_{\Omega} \Phi\left(u_{0}(x)\right) d x-\int_{\Omega} \Phi(u(x, T)) d x .
$$

Since $\lim _{T \rightarrow \infty} \int_{\Omega} \Phi(u(x, T)) d x$ exists, it follows that

$$
\nu \int_{0}^{\infty} \int_{\Omega}\left(u_{t}-f(u)\right)^{2} d x d t \leq C .
$$

Here and in what follows, $C$ will denote a generic constant independent of $t$. Now, we will obtain (4.2) if we prove that $h(t):=\int_{\Omega}\left(u_{t}(x, t)-f(u(x, t))\right)^{2} d x$ is uniformly continuous in $t \in[0, \infty)$.

Since $\|u(\cdot, t)\|_{L_{\infty}}$ is uniformly bounded, it follows from

$$
u_{t}-f(u)=\lambda^{-1}\left((I-\lambda \Delta)^{-1}-I\right) \phi(u),
$$

that $\left\|u_{t}(\cdot, t)-f(u(\cdot, t))\right\|_{L_{\infty}}$ is uniformly bounded.

For $t>s \geq 0$, we have

$$
\begin{aligned}
|h(t)-h(s)| & =\left|\int_{\Omega}\left(u_{t}(x, t)-f(u(x, t))\right)^{2}-\left(u_{t}(x, s)-f(u(x, s))\right)^{2} d x\right| \\
& \leq C \int_{\Omega}\left|u_{t}(x, t)-u_{t}(x, s)-(f(u(x, t))-f(u(x, s)))\right| d x \\
& =C \int_{\Omega}\left|\Delta(I-\lambda \Delta)^{-1}(\phi(u(x, t))-\phi(u(x, s)))\right| d x \\
& \leq C\|\phi(u(\cdot, t))-\phi(u(\cdot, s))\|_{L_{\infty}} \\
& \leq C\|u(\cdot, t)-u(\cdot, s)\|_{L_{\infty}} \\
& \leq C(t-s) .
\end{aligned}
$$

This proves that $h(t)$ is uniformly Lipschitz continuous, and (4.2) follows.

Interpolating between $L_{2}$ and $L_{\infty}$, we obtain that

$$
\left\|u_{t}-f(u)\right\|_{L_{p}} \rightarrow 0 \text { as } t \rightarrow \infty \text { for } 2 \leq p<\infty .
$$


To obtain (4.3), we observe that $J(\cdot, t)-\phi_{p r}(t)$ is the unique solution of the Neuman problem

$$
\Delta w(x)=u_{t}(x, t)-f(u(x, t)) \text { in } \Omega \text { with } \eta \cdot \nabla w=0 \text { in } \partial \Omega .
$$

Hence $\int_{\Omega} w=0$. This implies that for all $p$ such that $1<p<\infty$, there exists a constant $C$ such that

$$
\left\|J(\cdot, t)-\phi_{p r}(t)\right\|_{W^{2, p}} \leq C\left\|u_{t}-f(u)\right\|_{L_{p}} .
$$

When $2 p>d$, it follows that $W^{2, p}$ is continuously embedded in $L_{\infty}$. This implies (4.3).

Lemma 12. Under the hypotheses of Theorem 10

$$
\lim _{t \rightarrow \infty} \phi_{p r}(t) \text { exists. }
$$

Proof. Suppose that the limit does not exist. Choose $p$ and $q$ such that

$$
\liminf _{t \rightarrow \infty} \phi_{p r}(t)<p<q<\limsup _{t \rightarrow \infty} \phi_{p r}(t) .
$$

Hence, $0<p<q<\phi_{+}$. Recall the definition of $u_{i}(r)$ in Hypothesis 2. We choose $\epsilon>0$ small enough such that $\left|u_{1}(r)-u_{2}(r)\right|>5 \epsilon$ if $r \in[p, q]$. Let

$$
Q:=\left\{t: \phi_{p r}(t) \in[p, q]\right\}
$$

Let us define, for $t \in Q, i=1,2$,

$$
\begin{aligned}
S_{i}^{\epsilon}(t) & :=\left\{x \in \Omega:\left|u(x, t)-u_{i}\left(\phi_{p r}(t)\right)\right|<\epsilon\right\}, \\
\mu_{i}^{\epsilon}(t) & :=\left|S_{i}^{\epsilon}(t)\right|, \text { the measure of } S_{i}^{\epsilon}(t), \\
S_{0}^{\epsilon}(t) & :=\Omega \backslash \bigcup_{i=1}^{2} S_{i}^{\epsilon}(t), \\
\mu_{0}^{\epsilon}(t) & :=\left|S_{0}^{\epsilon}(t)\right| .
\end{aligned}
$$

The sets $S_{i}^{\epsilon}(t), i=0,1,2$, are disjoint. To prove Lemma 12 we need three sublemmas.

\section{Sublemma 1.}

$$
\lim _{t \rightarrow \infty, t \in Q} \sum_{i=1}^{2} \mu_{i}^{\epsilon}(t)=|\Omega| .
$$

Proof. It follows from Lemma 11 that the set

$$
S_{f}^{\delta}(t):=\left\{x \in \Omega:\left|\phi(u(x, t))-\phi_{p r}(t)\right|>\delta\right\}
$$

satisfies $\left|S_{f}^{\delta}(t)\right| \rightarrow 0$ as $t \rightarrow \infty, t \in Q$, for all $\delta>0$. Since $\phi$ is strictly monotonic in each of the subsets $\left\{u_{i}(r): r \in[p, q]\right\}, i=1,2$, we can choose $\delta>0$ such that $\left|u-u_{i}(r)\right|>\epsilon$ implies $|\phi(u)-r|>\delta$ for $r \in[p, q]$. Hence $S_{0}^{\epsilon}(t) \subset S_{f}^{\delta}(t)$, and we obtain the lemma.

Sublemma 2. For a given function $h \in C^{1}(\mathbb{R})$ with support supp $h \subset(0, \infty)$, we define $H(z):=\int_{0}^{z} h(\phi(s)) d s$. Hence,

$$
\lim _{t \rightarrow \infty} \int_{\Omega} H(u(x, t)) d x=\lim _{t \rightarrow \infty, t \in Q} \sum_{i=1}^{2} \mu_{i}^{\epsilon}(t) H\left(u_{i}\left(\phi_{p r}(t)\right)\right) .
$$

In particular, the limit on the right hand side exists. 
Proof. From Lemma 9 the limit on the left hand side exists. We write

$$
\begin{aligned}
\int_{\Omega} H(u(x, t)) d x= & \int_{S_{0}^{\epsilon}(t)} H(u)+\sum_{i=1}^{2} \int_{S_{i}^{\epsilon}(t)} H(u)-H\left(u_{i}\left(\phi_{p r}(t)\right)\right) \\
& +\sum_{i=1}^{2} \int_{S_{i}^{\epsilon}(t)} H\left(u_{i}\left(\phi_{p r}(t)\right)\right) \\
:= & T_{0}(t)+\sum_{i=1}^{2} T_{i}(t)+\sum_{i=1}^{2} \mu_{i}^{\epsilon}(t) H\left(u_{i}\left(\phi_{p r}(t)\right)\right) .
\end{aligned}
$$

Now, $\left|T_{0}(t)\right| \leq C \mu_{0}^{\epsilon}(t) \rightarrow 0$ as $t \rightarrow \infty$ in $Q$ (note that since supp $h \subset(0, \infty), H(u)$ is uniformly bounded).

For $i=1,2$

$$
\left|T_{i}(t)\right| \leq C \int_{S_{i}^{\epsilon}(t)}\left|u(x, t)-u_{i}\left(\phi_{p r}(t)\right)\right| d x
$$

where $C=\|h(\phi(\cdot))\|_{L_{\infty}(\mathbb{R})}$. For all $\delta, 0<\delta<\epsilon$, we have $S_{i}^{\delta}(t) \subset S_{i}^{\epsilon}(t)$, therefore

$$
\left|T_{i}(t)\right| \leq C\left(\delta \mu_{i}^{\delta}(t)+\epsilon\left(\mu_{i}^{\epsilon}(t)-\mu_{i}^{\delta}(t)\right)\right) .
$$

Adding this inequality over $i=1,2$, and using Sublemma 1, we obtain

$$
\limsup _{t \rightarrow \infty, t \in Q} \sum_{i=1}^{2}\left|T_{i}(t)\right| \leq C \delta, \text { for all } \delta>0 .
$$

Hence these terms converge to zero, and we obtain Sublemma 2 .

Sublemma 3. For each $i=1,2, \mu_{i}:=\lim _{t \rightarrow \infty, t \in Q} \mu_{i}^{\epsilon}(t)$ exists.

Proof. Let us choose two nonnegative $C^{1}$ functions $h_{-}(r), h_{+}(r)$ such that their supports satisfy supp $h_{-} \subset(0, p)$, supp $h_{+} \subset\left(q, \phi_{+}\right)$. Let

$$
H_{ \pm}:=\int_{0}^{z} h_{ \pm}(\phi(s)) d s
$$

For $t \in Q$ we can see that $H_{ \pm}\left(u_{i}\left(\phi_{p r}(t)\right)\right)$ is independent of $t$. In fact, for $r \in[p, q]$ there exist positive constants $\gamma_{i}^{ \pm}, i=1,2$, independent of $r$, such that

$$
\begin{aligned}
& H_{-}\left(u_{1}(r)\right)=\gamma_{1}^{-}, \\
& H_{-}\left(u_{2}(r)\right)=\gamma_{1}^{-}, \\
& H_{+}\left(u_{1}(r)\right)=0, \\
& H_{+}\left(u_{2}(r)\right)=\gamma_{1}^{+}+h_{2}^{+},
\end{aligned}
$$

where

$$
\begin{aligned}
\gamma_{1}^{-} & =\int_{0}^{u_{1}(p)} h_{-}(\phi(s)) d s, \\
\gamma_{1}^{+} & =\int_{u_{1}(q)}^{\alpha_{+}} h_{+}(\phi(s)) d s, \\
\gamma_{2}^{+} & =\int_{\alpha_{+}}^{u_{2}(q)} h_{+}(\phi(s)) d s .
\end{aligned}
$$


Applying Sublemmas 1 and 2 we find that

$$
\left(\begin{array}{cc}
\gamma_{1}^{-} & \gamma_{1}^{-} \\
0 & \gamma_{1}^{+}+\gamma_{2}^{+}
\end{array}\right)\left(\begin{array}{c}
\mu_{1}^{\epsilon}(t) \\
\mu_{2}^{\epsilon}(t)
\end{array}\right)=\left(\begin{array}{c}
H_{-}^{\infty} \\
H_{+}^{\infty}
\end{array}\right)+o(1)
$$

as $t \rightarrow \infty$ in $Q$, where $H_{ \pm}^{\infty}:=\lim _{t \rightarrow \infty} \int_{\Omega} H_{ \pm}(u(x, t)) d x$. The matrix in (4.6) is not singular, and from this we obtain Sublemma 3

Now, we are ready to prove Lemma 12. From Sublemmas 3 and 2 we have that for all $H$ as in Sublemma 2 .

$$
\lim _{t \rightarrow \infty} \int_{\Omega} H(u(x, t)) d x=\lim _{t \rightarrow \infty, t \in Q} \sum_{i=1}^{2} \mu_{i} H\left(u_{i}\left(\phi_{p r}(t)\right)\right) .
$$

In particular, the right hand side must be equal to

$$
\sum_{i=1}^{2} \mu_{i} H\left(u_{i}(r)\right) \text { for all } r \in[p, q] \text {. }
$$

Indeed, suppose that there exist $t$ and $s$ in $Q$ such that

$$
\sum_{i=1}^{2} \mu_{i} H\left(u_{i}\left(\phi_{p r}(t)\right)\right) \neq \sum_{i=1}^{2} \mu_{i} H\left(u_{i}\left(\phi_{p r}(s)\right)\right) .
$$

Hence, from (4.5) and since $\phi_{p r} \in C([0, \infty), \mathbb{R})$ (note that $u \in C\left([0, \infty), L_{\infty}(\Omega)\right.$ ) and $\phi$ is uniformly continuous), there exist sequences $t_{n}, s_{n} \in Q$ such that $t_{n}, s_{n} \rightarrow \infty$ as $n \rightarrow \infty$, and $\phi_{p r}\left(t_{n}\right)=\phi_{p r}(t), \phi_{p r}\left(s_{n}\right)=\phi_{p r}(s)$. This is a contradiction to the fact that the previous limit exists.

Let $h_{n}(z)$ be a sequence of $C^{1}$ functions such that $h_{n}(z) \equiv 1$ for $z \geq \phi\left(\frac{1}{n}\right)$ and supp $h_{n} \subset(0, \infty)$. Hence, for $n$ big enough we have

$$
\begin{aligned}
\lim _{t \rightarrow \infty} \int_{\Omega} H_{n}(u(x, t)) d x & =\sum_{i=1}^{2} \mu_{i} H_{n}\left(u_{i}(r)\right) \\
& =\sum_{i=1}^{2} \mu_{i}\left(\int_{0}^{\frac{1}{n}} h_{n}(\phi(z)) d z+\int_{\frac{1}{n}}^{u_{i}(r)} h_{n}(\phi(z)) d z\right) \\
& =\sum_{i=1}^{2} \mu_{i} u_{i}(r)+\left(\int_{0}^{\frac{1}{n}} h_{n}(\phi(z)) d z-\frac{1}{n}\right)|\Omega| .
\end{aligned}
$$

Therefore,

$$
\mu_{0}:=\lim _{n \rightarrow \infty} \lim _{t \rightarrow \infty} \int_{\Omega} H_{n}(u(x, t)) d x=\sum_{i=1}^{2} \mu_{i} u_{i}(r)
$$

for all $r \in[p, q]$. If $\phi$ satisfies Hypothesis 2, this takes us to a contradiction, and the proof of Lemma 4.3 is finished.

To finish the proof of Theorem 10, we recall that for almost every $x \in \Omega$, the function $t \mapsto u(x, t)$ is $C^{1}$ and satisfies $\lambda u_{t}=\lambda f(u)-\phi(u)+J(x, t)$. Let $\phi_{\infty}=$ $\lim _{t \rightarrow \infty} \phi_{p r}(t)$. From Lemmas 11 and 12 we obtain

$$
\lambda u_{t}=-\left(\phi(u)-\lambda f(u)-\phi_{\infty}\right)+e(x, t)
$$

where $|e(x, t)| \rightarrow 0$ as $t \rightarrow \infty$ uniformly for $x \in \Omega$. By the following lemma, proved in [13, Lemma 6], and Proposition 6], we obtain that $u(x, t)$ converges almost everywhere to a function $u_{\infty}: \Omega \rightarrow[0, \infty]$, and $\phi\left(u_{\infty}(x)\right)-\lambda f\left(u_{\infty}(x)\right)=\phi_{\infty}$ almost everywhere. From equation (4.7) we also obtain that $u_{t}(x, t) \rightarrow 0$ almost 
everywhere as $t \rightarrow \infty$. Therefore, from Lemma 11 we obtain that $f\left(u_{\infty}(x)\right)=$ $\lim _{t \rightarrow \infty} f(u(x, t))=0$ almost everywhere. This allows us to conclude that $u_{\infty}$ satisfies $\phi\left(u_{\infty}(x)\right)=\phi_{\infty}$ and $f\left(u_{\infty}(x)\right)=0$ almost everywhere, and the proof of Theorem 10 is finished.

Lemma 13. Suppose that $g: \mathbb{R} \rightarrow \mathbb{R}$ is continuous and not identically zero on any open interval. Suppose that $z(t) \in C^{1}(0, \infty)$ is a solution of the equation

$$
z^{\prime}(t)=g(z(t))+e(t)
$$

where $e(t)$ is continuous and $\lim _{t \rightarrow \infty} e(t)=0$. Then, $\lim _{t \rightarrow \infty} z(t)=z_{\infty}$ exists, and if $z_{\infty}$ is finite, $g\left(z_{\infty}\right)=0$.

The next result proves that the discontinuous steady state solutions of (1.1)-(1.2) may be a limit of continuous initial data $u_{0}$; by Proposition 5 we know that this initial data produce continuous solutions. Nevertheless, the convergence cannot be uniform since in a small $L_{\infty}$ neighborhood of a discontinuous solution there exist no continuous functions.

Proposition 14. Let $u_{0} \in L_{\infty}(\Omega)$ be such that $u_{0}(x) \geq 0$. Then, for all $x \in \Omega^{*}$ we have

$$
\begin{array}{ll}
\text { If } & u_{0}(x)<\alpha, \quad \text { then } u(x, t)<\alpha \quad \text { for all } t \geq 0 . \\
\text { If } \quad u_{0}(x)>a, \quad \text { then } u(x, t)>a & \text { for all } t \geq 0 .
\end{array}
$$

Therefore $u_{\infty}(x):=\lim _{t \rightarrow \infty} u(x, t)$, which exists by Theorem 10, is a stationary solution of (1.1) - (1.2) and for all $x \in \Omega^{*}$

$$
\begin{array}{ll}
\text { If } \quad u_{0}(x)<\alpha, \text { then } u_{\infty}(x)=0 . \\
\text { If } u_{0}(x)>a, \text { then } u_{\infty}(x)=b .
\end{array}
$$

Proof. The proof is similar to the proof of Proposition 6 and is omitted.

Remark 4. As a consequence of this result we obtain that problem (1.1)-(1.2), with $\beta=a$ and $\phi(u)=0$ for $u \geq a$, exhibits permanent recovery. Certainly, from Proposition 8 we have that problem (1.1)-(1.2) exhibits recovery. Since Proposition 14 implies that if $u\left(x, t_{0}\right)>a$ then $u(x, t)>a$ for all $t \geq t_{0}$, the recovery is permanent. Moreover, by the proof of Proposition 8 , there exist a proper subset $\Omega_{0}$ of $\Omega$ with positive measure and $t_{0}>0$ such that $u\left(x, t_{0}\right)>a$ for almost every $x \in \Omega_{0}$ and $u\left(x, t_{0}\right)<\alpha$ for almost every $x \in \Omega \backslash \Omega_{0}$. By Theorem 10 and Proposition 14 we have that $\lim _{t \rightarrow \infty} u(x, t)=u_{\infty}(x)$ where

$$
u_{\infty}(x)= \begin{cases}b & \text { if } x \in \Omega_{0}, \\ 0 & \text { if } x \in \Omega \backslash \Omega_{0},\end{cases}
$$

is an equilibrium solution of (1.1) $-(1.2)$.

\section{REFERENCES}

1. W.C. Allee, Animal aggregations, University of Chicago Press, Chicago, 1931.

2. D.G. Aronson, The role of diffusion in mathematical population biology: Skellam revisited, in "Mathematics in Biology and Medicine", Lecture Notes in Biomathematics 57, S. Levin, Springer-Verlag, Berlin,1985. MR 86g:92002

3. D.G. Aronson, Density-dependent interaction-difusion systems, in "Dynamics and Modeling of Reactive Systems", W.E. Stewart et al., Acad. Press, 1980. MR 82a:35056

4. J. Dieudonné, Foundations of Modern Analysis, Academic Press, New York and London, 1960. MR 22:11074

5. P. Grindrod, Models of individual aggregation in single and multispecies communities, J. Math. Biol. 26 (1988), 651-660. MR 90a:92041] 
6. D. Grunbaum and A. Okubo, Modeling social animal aggregations, in "Frontiers in Mathematical Biology", Lecture Notes in Biomathematics 100, S.A. Levin, Springer-Verlag, New York, 1994. MR 96d:92001

7. M.E. Gurtin and R.C. MacCamy, On the diffusion of biological populations, Mathematical biosciences 33 (1977), 35-49. MR 58:33147]

8. M.A. Lewis and P. Kareiva, Allee dynamics and the spread of invading organisms, Theoretical Population Biology 43 (1993), 141-158.

9. M. Lizana and V. Padrón, A spatially discrete model for aggregating populations, J. Math. Biol. 38 (1999), 79-102. MR 99m:92034

10. J.D. Murray, Mathematical Biology, Biomathematics 19, Springer-Verlag, 1993. MR 94j:92002

11. A. Novick-Cohen and R.L. Pego, Stable patterns in a viscous diffusion equation, Trans. Amer. Math. Soc. 324 (1991), 331-351. MR 91f:35152

12. A. Okubo, Diffusion and Ecological Problems: Mathematical Models, Biomathematics 10, Springer-Verlag, New York, 1980. MR 81i:92025

13. V. Padrón, Sobolev regularization of a nonlinear ill-posed parabolic problem as a model for aggregating populations, Commun. in Partial Differential Equations 23 (1998), 457-486. MR 99k:35185

14. M. Protter and H. Weinberger, Maximum principles in differential equations, Springer-Verlag, New York, 1984. MR 86f:35034

15. J.G. Skellam, Random dispersal in theoretical populations, Biometrika 38 (1951), 196-218. MR 13:263b

16. J.G. Skellam, The formulation and interpretation of mathematical models of diffusionary processes in population biology, in "The Mathematical Theory of the Dynamics of Biological Populations", M.S. Bartlett and R.W. Hiorns, eds., Acad. Press, New York, 1973. MR 58:20591a

Facultad de Ciencias, Departamento de Matemáticas, Universidad de Los Andes, MÉrida 5101, Venezuela

E-mail address: padron@ula.ve 